\title{
El consentimiento informado en Colombia. Un análisis comparativo del proyecto de ley 24 de 2015 con el código vigente y otros códigos de ética
}

Consent informed in Colombia:

A comparative analysis of the proposed law 24 of 2015 with the current code and other codes of ethics

\author{
Juliana Maria Mendoza Villa ${ }^{1 \boldsymbol{*}}$, Luis Andrés Herrera Morales
}

Fecha correspondencia:

Recibido: febrero 2 de 2017.

Revisión: febrero 17 de 2017.

Aceptado: marzo 28 de 2017.

Forma de citar:

Mendoza, J., Herrera, L. (2017). El

consentimiento informado en Colombia. Un

análisis comparativo del proyecto de ley 24 de

2015 con el código vigente y otros códigos de

ética. Rev. CES Derecho., 8(1), 156-171.

Open access

Términos de uso

Licencia creative commons

Ética de publicaciones

Revisión por pares

Gestión por Open Journal System

DOI: http://dx.doi.org/10.21615/cesder.8.1.8

ISSN: 2145-7719

Sobre los autores:

1. Médica, anestesióloga de ANESTESIAR

Sindicato, profesora asociada de

anestesiología, Miembro de Junta Directiva

de Sociedad Colombiana de Anestesiología

y Reanimación - S.C.A.R.E, y aspirante a

magister en bioética de Universidad CES

2. Médico, anestesiólogo de ANESTESIAR

Sindicato, profesor asociado de anestesiología,

\section{Comparte}

\section{Resumen}

Cursa en el Congreso colombiano un proyecto de ley para renovar el Código de Ética Médica (Proyecto de ley 24 de 2015). Este proyecto, enraizado en el reconocimiento del principio de libertad moral, proclama el respeto por la autonomía del paciente como uno de los constituyentes de la ética asistencial moderna.

Ante la valiosa iniciativa se revisó lo atinente al consentimiento informado (Cl) y se efectuó un análisis comparativo con el código vigente (la Ley 23 de 1981) y otros contemporáneos (el chileno, el español y el argentino), en busca de fortalezas y debilidades.

Se encontró que el proyecto de ley no cuenta con los contenidos filosóficos, bioéticos y procedimentales suficientes para cumplir con su promesa de valor. En cambio, se caracteriza, por ser extenso en temas sancionatorios. Tal desequilibrio podría derivar en una práctica médica más defensiva y menos ética.

Palabras clave: Consentimiento informado; bioética; legislación; autonomía; autonomía profesional; práctica clínica.

\section{Abstract}

The Colombian Congress is currently debating a bill to renew the Code of Medical Ethics (Bill 24/2015). This bill, rooted in a recognition of the principle of moral freedom, proclaims respect for a patient's personal autonomy as one of the most important components of modern clinical ethics.

In light of this valuable initiative, the researchers reviewed topics related to informed consent and undertook a comparative analysis between the current Code (Law 23/1981) and other contemporary laws (from Chile, Spain and Argentina), looking for their strengths and weaknesses.

The authors found that the bill lacks the philosophical, bioethical and procedural content required to fulfill its promises. Rather, it appears to emphasize punitive topics and sanctions, and this imbalance could result in a more defensive and less ethical medical practice. 
aspirante a magister en bioética de la Universidad CES, Coordinador de anestesia Clínica del Norte (Bello, Antioquia,

Colombia); Vicepresidente de la Sociedad Antioqueña de Anestesia - SADEA.
Keywords: Informed consent; ethics; legislation; personal autonomy; professional autonomy; practice patterns, physicians.

\section{Introducción}

La ética clásica forjó al paternalismo como el paradigma de la atención en salud (Gracia, 2004b). Hoy el ejercicio médico ha cambiado a tal punto que ser paternalista es considerado moralmente incorrecto (Gracia, 2004a) y a tal extremo que la inclusión de los pacientes en el proceso de toma de decisiones parece confundirse con la subrogación del criterio clínico. A su vez, la acelerada tecnificación de la medicina y el vertiginoso contexto social en el que esta se mueve precipitan dilemas éticos cada vez más complejos que reclaman soluciones.

Colombia no es ajena a tal contexto. El país que fundamentó la Ley 23 de 1981 y que en los últimos 35 años ha estrenado Constitución Política, Sistema de Seguridad Social y Ley Estatutaria en Salud, también ha experimentado significativas transformaciones culturales y mutaciones en su acervo que deviene en nuevas obligaciones sociales. En consecuencia, el Congreso colombiano se encuentra debatiendo un proyecto para promulgar un nuevo Código de Ética Médica, el cual pretende superar el paternalismo y servir como un instrumento que posibilite el desarrollo de una relación clara, justa y fluida entre el trinomio paciente-médico-Estado, acorde con las demandas de la sociedad y del ejercicio médico contemporáneo.

El presente artículo pretende estudiar el contenido del proyecto en lo referente al consentimiento informado -en adelante $\mathrm{Cl}$-, comparándolo con otros códigos de ética médica. Se escoge el $\mathrm{Cl}$ como un eje central de la discusión porque representa la manifestación de la autonomía del paciente y una expresión de respeto hacia este (Kottow, 2007).

\section{Análisis del contexto jurídico colombiano.}

Fundamentado en la evolución moderna del concepto de autonomía - "autós": uno mismo; "nomos": ley-; partiendo desde el primer código que establecía criterios para regular la investigación en seres humanos -Alemania, 1931-; pasando por el Código de Núremberg -1-947, por la declaración de Helsinki -1964 a 2013-y por el informe Belmont -1978-; el Cl constituye hoy un elemento esencial en la relación médico-paciente (Cañete, Guilhem y Brito, 2012), ahora también llamada relación equipo de salud-usuario (Ocampo, 2002).

En Colombia, la Corte Constitucional y el Consejo de Estado han considerado el $\mathrm{Cl}$ como un desarrollo específico de varios derechos fundamentales ${ }^{1}$, siendo su médula la protección de la autonomía del paciente. También hace parte del deber de información que le es exigible al profesional, de hecho la jurisprudencia ha impuesto sanciones para indemnizar este derecho fundamental cuando lo ha considerado vulnerado.

Según la doctrina jurídica, se entiende el $\mathrm{Cl}$ como un proceso de ilustración continuada al paciente, en el cual éste expresa su voluntad de aceptar o rechazar un plan diagnóstico, terapéutico o investigativo- formulado por el médico. Este proceso debe permitir que el paciente comprenda los principales beneficios, riesgos y alternativas

1 La jurisprudencia en Colombia al respecto es amplia. Ver, entre otras, las sentencias: C221/94; T-401/94; T411/94; T-477/95; T-474/96; SU-337/99; T-1390/00; T-1390/00; T-597/01; T-823/02; T-850/02; T-866/06; T-1025/02; T-1021/03; T-510/03; T-762/04; T-1131/04; T-1229/05; T-866/06; T-1019/06; T-560A/07; T216/08; T653/08; T-921/08; T-452/10; T-622/14; C-182/16. Sentencias del Consejo de Estado, Sección Tercera: 9 de julio de 1993, exp.7795; 3 de mayo de 1999, exp.11169; 24 de enero del 2002 exp.12706; 13 de diciembre de 2004, exp.14722; 3 de mayo del 2007, exp.16098; 23 de abril de 2008, exp.15737 y del 27 de marzo del 2014, exp.26660. En este mismo sentido, ver Corte Suprema de Justicia, Sala de Casación Civil, sentencia del 27 de julio de 2015, rad. SC9721-2015, p.50-53 
disponibles a la propuesta dada, para que, en uso de su derecho a la autodeterminación y al libre desarrollo de su personalidad, pueda contar con los suficientes elementos de valor que le permitan decidir sobre las actuaciones que incidirán en su salud.

Para su ejecución, el médico debe partir del supuesto de la ignorancia del paciente en la materia. Además, debe ponderar un conjunto de factores como²: el grado de invasividad del tratamiento, la urgencia del mismo, los riesgos derivados de su demora, su estado de aceptación y evidencia clínica, su nivel de dificultad, las probabilidades de éxito, la afectación de derechos e intereses personales del sujeto al efectuarse el tratamiento, la afectación de derechos de terceros de no realizarse la intervención, la capacidad de comprensión del sujeto acerca de los efectos directos y colaterales del tratamiento sobre su persona, la existencia de alternativas terapéuticas y la posible renuncia del paciente a recibir información.

La doctrina nacional también destaca como responsabilidad del médico el deber de considerar la individualidad del paciente; sin embargo, la complejidad de esta presunción se demuestra en su falta de precisión. A la exigencia de brindar una información suficiente, oportuna, simple, aproximativa, razonable, equilibrada, clara, precisa y leal, se le suma la de graduar la forma y el contenido de la misma, ponderando la capacidad de compresión del paciente y su posible impacto psicológico negativo ${ }^{3}-4$. Tal alcance es ilusorio, pues se mueve en el terreno de lo intersubjetivo y de lo muy íntimo, más aún desde la presunción de ser una obligación y responsabilidad exclusiva del médico.

Así, el ejercicio de la profesión médica camina sobre un hilo muy delgado, y en principio contradictorio, entre sus deberes, sus capacidades y las condiciones y limitaciones del sistema que lo sustenta. Para dar otro ejemplo, el tiempo destinado para el proceso de información, que valga precisar es ínfimo, no suele ser optativo del profesional pues está sujeto a políticas institucionales de atención, y estas, a su vez, están derivadas de conceptos de rentabilidad económica sin un adecuado equilibrio con la rentabilidad social. Además, la segmentación de las funciones asistenciales en salud, que ha sido producto de la emergencia de múltiples disciplinas y de la subespecialización, exige una dinámica que permita integrar la información, lo cual, requeriría cambiar el paradigma de acción netamente individual del profesional por un engranaje de equipos de trabajo y de procesos médico-institucionales.

Incompatibilidades similares plantea la Ley 23 de $1981^{5}$. Por ejemplo, en su Declaración de Principios postula el "perfeccionamiento de la especie humana" como uno de los fines de la medicina. Este concepto, que parece partir de la filosofía nietzschea-

2 Cfr. la sentencia T-1131/04: "Sólo a partir de una ponderada combinación de los anteriores elementos puede cualificarse, en cada caso concreto, el nivel de información que requiere el paciente para adoptar la decisión autónoma de someterse a una intervención médica."

3 Véase la sentencia T1131/04 de la Corte Constitucional, que establece el principio de cautela como un deber: "El médico deberá, en uso de su privilegio terapéutico, proporcionar la información que no genere en el paciente un aumento desproporcionado de su angustia, pues una información no medida en los factores señalados, puede dañar más al enfermo que beneficiarlo". Sin embargo, se ha reconocido la dificultad del ejercicio de esta obligación en la Sentencia SU-337/99, que señala: "No es pues posible, dada la complejidad de los casos concretos, formular unas reglas rígidas sobre el alcance de la información que debe ser suministrada por los médicos. Tan solo se puede establecer una pauta, como la señalada en el fundamento jurídico 14 de esta sentencia, la cual permite evaluar, dadas las particularidades de las distintas situaciones, si los profesionales de la salud han cumplido o no con su obligación de información."

4 En este sentido debe tenerse en cuenta lo establecido en el decreto 3380 de 1981, mediante el cual se reglamentó la ley 23 de 1981 que establece que el "médico cumple la advertencia del riesgo previsto, a que se refiere el inciso segundo del artículo 16 de la ley 23 de 1981, con el aviso que en forma prudente, haga a su paciente o a sus familiares o allegados, con respecto a los efectos adversos que, en su concepto, dentro del campo de la práctica médica, puede llegar a producirse como consecuencia del tratamiento o procedimiento médico"

5 Cfr. Código Colombiano de Ética Médica, Congreso de la República de Colombia, 1981, p. 23. Decreto Reglamentario, 3380 de noviembre 30 de 1981, artículos 11 y 12. 
na, contradice el carácter personalísimo de la profesión, el cual está fundamentado en el respeto por la diversidad y la particularidad del ser humano, a su vez integrado en los conceptos de autonomía-vulnerabilidad y dignidad de cada individuo; y ejemplifica las incongruencias que pueden existir entre lo jurídico y lo ético.

En términos generales, el Código de ética médica vigente es amplio en los procedimientos sancionatorios y de funcionamiento de los entes punitivos, pero insuficiente en los temas deontológicos y bioéticos fundamentales; e innecesariamente diverso en consideraciones triviales como la estética que debe tener el consultorio del médico. Esta visión reduce la responsabilidad moral a la mera responsabilidad jurídica, y olvida la importancia práctica que tienen estos códigos al servir como guía moral del profesional y delinear la identidad del profesional que la sociedad desea.

Además, la Ley 23 carece de normatividad para guiar el ejercicio de los administradores sanitarios, quienes, sin duda, hoy son actores protagónicos en la prestación de servicios. Si bien, el objeto de esta ley no definió dentro de su alcance la regulación de los deberes de otros actores del sistema de salud, la realidad actual hace imperioso que se analice el papel y las responsabilidades de quienes ejercen como administradores, bien sea de instituciones o de cualquier otro proceso que tenga injerencia en decisiones que impacten en la atención de los pacientes y, por lo mismo, en aspectos inescindiblemente ligados con el actuar médico. Estas decisiones que generalmente se denominan "administrativas" influyen de manera directa en los servicios que se prestan al usuario. Si bien existen normas al respecto, por ejemplo para los prestadores, estas no están unificadas en un solo cuerpo normativo con las correspondientes sanciones.

En relación con el tema del $\mathrm{Cl}$, la norma vigente parte de la suspicacia sobre el criterio clínico, es exigua en la promoción de la autonomía del paciente y da un carácter fáctico al acto médico. ${ }^{6}$ Tampoco establece pautas concretas que sirvan para guiar la conducta del profesional en casos especiales como, por ejemplo, cómo actuar ante pacientes menores de edad, en casos de conflictos de interés entre representantes legales o frente a conflictos éticos del final de la vida, entre otros. Probablemente, para el momento en que fue redactada, no se contemplaron la diversidad de dilemas bioéticos que la Medicina moderna encarna, algunos de los cuales han sido tratados ya en los tribunales.

\section{Proyecto de Ley 24 de 2015}

La introducción del Proyecto (Senado de la República de Colombia, 2016) es acertada en la justificación filosófica y bioética sobre el porqué se requiere formular un nuevo código de ética médica. Desde sus fundamentos intenta separarse del paradigma precedente y esboza el paso de una relación médico-paciente a una relación médico-paciente-Estado ${ }^{7}$. Asimismo, acierta en identificar como "obligaciones de medios" al tipo de compromisos que surgen del acto médico ${ }^{8}$.

6 Cabe aclarar que el código de ética médica colombiano y el proyecto de su reforma -a diferencia otros códigos- no fueron expedidos por el Colegio Médico del país, son leyes promulgadas por el Congreso de la República. De los 58 artículos exequibles a la fecha, pocos tratan el tema del Cl. Específicamente, en el capítulo I, los artículos 15 y 16 señalan: "El médico no expondrá a su paciente a riesgos injustificados. Pedirá su consentimiento para aplicar tratamientos (...) que considere indispensables y que puedan afectarlo física o síquicamente (...) y le explicará al paciente o a su responsable de tales consecuencias anticipadamente". Con relación a esto la Corte Suprema de Justicia ha dicho: "Así, es la omisión de la obligación de informar y obtener el consentimiento informado la que hace responsable al médico y, por consiguiente, a las instituciones prestadoras del servicio de salud" (Corte Suprema de Justicia, Sala de Casación Civil, M.P. Fernando Giraldo Gutiérrez. Sentencia del 27 de julio de 2015 , rad. SC9721-2015).

7 "Junto con el de la autonomía, los principios morales de beneficencia y justicia constituyen el trípode que sirve de base de sustentación a la ética médica actual. El paternalismo registrado en el Juramento Hipocrático, que caracterizó a la medicina durante veinticinco siglos, dejó de tener vigencia. El paciente superó su condición de incapacitado mental y moral para convertirse en un sujeto activo, con derechos legales; el médico a su vez, continúa siendo el benefactor del paciente, pero no a contrapelo del querer de este; y finalmente el Estado, que en cuestiones médicas no fue tenido en cuenta, asumió la responsabilidad de estatuir la salud como un derecho y de garantizar su vigencia, es decir, de actuar con criterio justo" (Senado de la República de Colombia, 2015).

8 No sin controversia, este concepto ha previamente sido ratificado. Ver: Corte Suprema de Justicia, Sentencia del 5 de Marzo de 1940: Consejo de Estado, Sala de lo Contencioso Administrativo, Sección Tercera, Sentencia del 18 de Abril de 1994, expediente 
Empero, en su declaración de principios, la propuesta podría considerarse insuficiente para cumplir con lo que se dilucida son sus objetivos principales: servir de fundamento ético para regular el ejercicio de los profesionales titulados y de directriz hermenéutica en la interpretación e integración jurídica. Tal debilidad es esencial, pues, para poder sobrepasar su carácter de faro y abrazar las voluntades e intereses más legítimos de sus representados, al tratarse de una declaración de máximos morales, sus principios deben ser contundentes, coherentes y representativos del espíritu de la profesión médica.

A modo de ilustración, el Proyecto propone el principio de supervivencia, el cual, más que un postulado ético, es una capacidad o impulso vital, y está fuera del alcance de la deontología médica -siendo más del área de la ecología, la biología y la sociología-; deja por fuera principios primordiales como el de precaución, equidad, integridad, transparencia, honestidad y solidaridad; cae en impresiones que el discurso bioético ha resuelto, como mezclar los principios de beneficencia y de no maleficencia (Beauchamp \& Childress, 2001); confunde el concepto de justicia con el deber a una remuneración médica justa, que si bien es un tema muy importante, no constituye un valor de la medicina sino un derecho laboral.

De igual manera, presenta como principio al humanitarismo, el cual, como ideología o virtud humana, despliega nociones que sirven poco para guiar conductas morales concretas. Por ejemplo, entendiéndolo como el deber de promover el bienestar humano, en el contexto del ejercicio médico, tal valor queda perfectamente contenido por el principio de beneficencia. Comprendiéndolo como la compasión por las desgracias ajenas y el impulso de ayudar, se exporta la controversia que ha sido esbozada por quienes se apoyan en tal bandera. Fiona Terry, autora reconocida y con larga experiencia en trabajo humanitario, señala que la responsabilidad primordial por la seguridad y el bienestar de los ciudadanos incumbe a los Gobiernos, y los individuos no heredan, en principio, las responsabilidades morales que los Estados han eludido (Terry, 2002).

Ahora bien, para que su intención sea legítima y no caiga en una excusa para justificar el paternalismo, tal ideología debe estar provista de neutralidad, imparcialidad y responder a los intereses del paciente, volviendo a caer en tierra de la beneficencia. Finalmente, aceptando al humanitarismo como un impulso que mueve al hombre a crecer en conciencia, responsabilidad y sentido social, el concepto representa una característica filantrópica, una virtud propia de la diversidad humana, que no puede exigirse a todos y, por tanto, no puede proponerse como un imperativo jurídico.

En lo que al $\mathrm{Cl}$ se refiere, en su artículo 17 el Proyecto toca temas importantes que no son tratados por el código vigente, como el concepto de información suficiente, el consentimiento cualificado, la voluntad anticipada y la objeción de conciencia. Sin embargo, se aprecia insuficiente y ambiguo en su formulación, lo que podría afectar el desarrollo de la futura reglamentación. Por ejemplo, necesita proposiciones claras en cuanto al consentimiento sustituto; cómo se define el deber de cualificar y asistir el consentimiento en casos especiales"; qué se entiende por "cambios trascendentales en la vida del paciente", entre otros.

Además, se contradice en temas que se desligan de su fundamentación y de los precedentes normativos. El parágrafo 3, v.gr., señala: "El consentimiento informado es

7973; Sentencia T-313/96; Ley 1438 de 2011, artículo104; entre otros).

9 El parágrafo 2 del artículo 17 enuncia: "En los casos de procedimientos experimentales, extraordinarios, invasivos, de alto riesgo, o que puedan implicar cambios trascendentales en la vida del paciente, el consentimiento debe ser cualificado". Cfr. Corte Constitucional, Sentencias T-622/14 y C-900/11 
el marco general de autorización con que cuenta el facultativo, pero no se requiere su validación permanente para cada acto particular". Este parágrafo colisiona con el enunciado principal del artículo al que pertenece, el cual estipula la obtención del $\mathrm{Cl}$ "para la práctica de cualquier acto médico".

Probablemente lo que debe precisar el Proyecto para solventar tal ambigüedad es el concepto de proceso de consentimiento informado y los requisitos de su documentación probatoria. Sin embargo, en Colombia, la norma de habilitación para los servicios prestadores de salud es clara en exigir la manifestación documental del consentimiento del paciente para que tenga lugar el acto asistencial. ${ }^{10}$ Además, la jurisprudencia también ha interpretado como imprudentes los casos en donde el Cl está ausente en el contexto de tratamientos electivos, declarando la responsabilidad médica del acto al no poder comprobar que el paciente asumía unos riesgos conocidos. ${ }^{11}$

De igual modo, a pesar de que en su propuesta de valor desea integrar el concepto de relación paciente-médico-Estado, el Proyecto carece casi por completo de los criterios de responsabilidad ética que deben regir el ejercicio de los administradores sanitarios y demás entes que gobiernan el sistema de salud.

En conclusión, similar a la ley vigente, la iniciativa de ley es muy rica en lo punitivo casi a la par de un código penal-, pero necesita mayores desarrollos en sus contenidos bioéticos y procedimentales para hacerla útil al ejercicio profesional, pertinente y coherente con la actualidad normativa y la realidad social del país.

\section{El consentimiento informado en otros códigos de ética médica}

\section{Código de ética médica chileno}

El Código chileno se caracteriza por ser conservador y simple en sus definiciones (Colegio Médico Chileno, 2011). Es una regla genérica que evita dar lineamientos sobre dilemas éticos particulares.

Meritoriamente es el más categórico sobre la tortura con participación médica, hecho doloroso en su historia reciente.

Otra de las excepciones a su universalidad es en lo referente al Cl. En la sección De las Relaciones del Médico con sus Pacientes, el punto 2 contiene cinco artículos que abordan el tema del $\mathrm{Cl}$ e indican el tipo de información que se debe dar al paciente, la templanza requerida para transmitirla y el responsable de hacerlo.

Demarcando algunas diferencias con el código colombiano vigente y la iniciativa en curso, el código chileno abre espacio para el asentimiento informado en menores de edad $^{12}$. Además, empodera al paciente del manejo de su información, incluso para

10 Cfr. La Resolución 1441 del Ministerio de Salud de Colombia -"Por la cual se definen los procedimientos y condiciones que deben cumplir los Prestadores de Servicios de Salud para habilitar los servicios y se dictan otras disposiciones"- define el consentimiento informado así: "Es la aceptación libre, voluntaria y consciente de un paciente o usuario, manifestada en el pleno uso de sus facultades después de recibir la información adecuada, para que tenga lugar un acto asistencial. Para efectos del estándar de historia clínica es el documento que se produce luego de la aceptación en las condiciones descritas".

11 Asimismo, la Corte ha indicado que "todo tratamiento, aún el más elemental, debe hacerse con el consentimiento del paciente" Ver la Sentencia T-401 de 1994. En similar sentido, las sentencias T-823 de 2002, T-1021 de 2003, T-762 de 2004, T-1019 de 2006 y T-560 A de 2007.

12 El Artículo 27 del Código chileno señala: “La opinión del menor de edad deberá ser considerada, atendiendo a su edad y grado de madurez". En cuanto al Proyecto de ley colombiano, ha sido escindido este párrafo: "Cuando se trate de menores entre los siete (7) y los dieciocho (18) años de edad se obtendrá su asentimiento informado siempre que sea posible". Si bien la UNICEF menciona que el uso del asentimiento no es universalmente reconocido ni respaldado, por diversas circunstancias, también dice que asentimiento y consentimiento pueden coexistir y no tienen que ser mutuamente excluyentes (Graham A, Powell M, Taylor N, Anderson D, \& Fitzgerald R, 2013). Esto es respaldado en lo referente a los aspectos bioéticos y jurídicos del contexto colombiano (Pinto D \& Gulfo R, 2013). 
fines científicos. El facultativo tiene que obtener autorización escrita del paciente, o su representante, para revelar la identidad u otros aspectos. El permiso debe ser concreto en relación con el tipo y características de los datos a develar, y no se admite la validez de "autorizaciones genéricas, otorgadas con anterioridad a los hechos que las motivan, o insertas en contratos de adhesión celebrados con instituciones financiadoras o proveedoras de prestaciones de salud".

\section{Código de deontología médica español}

El Código de deontología médica español es una guía normativa (Organización Médica Colegial de España, 2011) -denominada así desde el título-, no una ley, y claramente busca diferenciarse de ello ${ }^{13}$. Es rico en fundamentos filosóficos, bioéticos y en aspectos instrumentales, como las razones por las cuales los médicos deben involucrarse en aspectos administrativos, entre otros. Al no ser sometido a trámites parlamentarios se facilita su revisión y actualización, hecho manifiesto de tal forma que, buscando acoplarlo a los vertiginosos avances de la Medicina y después de haber sido publicado en 1978, ha sido modificado ya en tres ocasiones -1990, 1999 y $2011-$.

Enmarcado en el respeto por la autonomía del facultativo, el Código establece la confianza como un valor necesario en la relación médico-paciente y su ausencia le permite al médico suspender de manera unilateral la asistencia. Además, atiende el asunto de la futilidad y la objeción de consciencia: "Si el paciente exigiera del médico un procedimiento que éste, por razones científicas o éticas, juzgase inadecuado o inaceptable, el médico, tras informarle debidamente, quedará dispensado de actuar".

En concordancia con esto, el Código le da preponderancia al proceso clínico del Cl, diferenciándolo de un acto burocrático. De esta manera, la expresión verbal del consentimiento tiene suficiente validez, siempre y cuando se deje constancia de ello en la historia clínica. Sólo define la necesidad de expedir un $\mathrm{Cl}$ por escrito en los casos que supongan un "riesgo significativo"14. A su vez, ofrece directrices específicas sobre cómo actuar ante menores de edad, por ejemplo, define que en los casos de pacientes menores de 16 años se requiere el consentimiento de los padres para las actuaciones que entrañen "riesgo grave", y la opinión del menor será determinante según su edad y grado de madurez, mientras que los pacientes mayores de 16 años se consideran en capacidad para tomar decisiones sobre acciones asistenciales ordinarias, sus padres deben ser informados y su opinión será tenida en cuenta, es decir, en estos casos son los menores los que consienten y sus padres asienten.

\section{Código de ética médica argentino}

La Asociación Médica Argentina, desde sus primeros estatutos, estableció que podían integrarla médicos y otros miembros del equipo sanitario (Asociación Médica Argentina, 2011). Así, reconoce la multiplicidad de actores que moldean la prestación de servicios en salud y, sobre esta base, manifiesta construir códigos incluyentes, dinámicos y adaptados a los avances de la sociedad. En concordancia, el Código de ética argentino esta creado para todo el equipo de salud y brinda observaciones específicas a médicos, enfermeros, farmacéuticos, bioquímicos, ingenieros, arquitectos hospitalarios, odontólogos, etcétera.

13 "Este Código sirve para confirmar el compromiso de la profesión médica con la sociedad a la que presta su servicio, incluyendo el avance de los conocimientos científico-técnicos y el desarrollo de nuevos derechos y responsabilidades de médicos y pacientes. Las pautas contenidas en él deben distinguirse de las imposiciones descritas en la ley". (Cfr. Preámbulo, p.7).

14 Sin precisar muy bien esta significado, el artículo 16 refiere: "El consentimiento se expresa habitualmente de forma verbal, dejando constancia en la historia clínica. Cuando las medidas propuestas supongan para el paciente un riesgo significativo se obtendrá el consentimiento por escrito". 
El Código define bien el alcance de las profesiones sanitarias y las aleja del ideal de la perfección:

El Miembro del Equipo de Salud en acción se compromete a utilizar todos los medios a su alcance para efectuar su prestación apoyándose en la formación de su predisposición de dar y cimentada en sus conocimientos y adiestramiento técnico-específico para prevenir, curar o aliviar los efectos de la enfermedad, en el marco de informar sobre los posibles riesgos y complicaciones inherentes a la misma, sin poder garantizar los resultados. ${ }^{15}$

Con relación a las responsabilidades del equipo sanitario, resalta el paso del paternalismo al respeto por la autonomía del paciente ${ }^{16}$. No es tan extenso en lo punitivo -versus los modelos colombianos-, más sí lo es en lo filosófico. Es el único que acertadamente principia definiendo el "ethos" y la ética", aunque aclara que "la ética no es una ciencia positiva" y por ello "no describe los actos humanos como son, sino como deben ser", por lo que es "una ciencia normativa".

Previendo las consecuencias del "ánimo penitenciario" de los legisladores el Código advierte: "Destacamos el reconocer que la palabra también es un agente agresor (...) Las leyes son palabras, por eso, cuando se debate una ley hay que participar por su repercusión futura. El legislador también es responsable en esta Cascada de Responsabilidades en Salud. Esto conduce a que se deba esclarecer a los legisladores, a sus asesores y a los funcionarios con poder de definición, para que comprendan la gravedad de una medicina sin responsabilidad como es la medicina defensiva." 18

En el artículo 22, configura una grave falta ética cuando el miembro del Equipo de Salud indica tratamientos sin la aclaración pertinente y el consentimiento previo del paciente o responsable.

También distingue entre el proceso de $\mathrm{Cl}$ y el documento atinente a ello, sin generar obligatoriedad del segundo en todos los casos. ${ }^{19}$ Además, establece recomendaciones específicas del $\mathrm{Cl}$ en situaciones especiales como: anticoncepción, trasplantes, cuidado del paciente adicto, paciente siquiátrico, paciente terminal, eutanasia, suicidio asistido, criopreservación y experimentación con humanos.

Singularmente, es el único código de los revisados que indica el $\mathrm{Cl}$ del paciente para la notificación de certificados con el diagnóstico. También es el único código que resuelve la duda que ocasionalmente se genera en los equipos sanitarios referente al $\mathrm{Cl}$ en los casos de trabajo en equipo. ${ }^{20}$ Además, protege a los pacientes en lo concerniente al manejo de los datos a través de historias clínicas electrónicas. ${ }^{21}$

15 Asociación Médica Argentina (2011). Código de Ética para el Equipo de Salud. Segunda edición. Página 21. 16 El Artículo 93 señala: "Los miembros del Equipo de Salud, aún aquellos con las más altas calificaciones de prestigio profesional y académico, deben evitar actitudes de condescendiente omnipotencia y paternalismo con los enfermos o sus familiares. Una disposición positiva para analizar en forma conjunta los problemas permitirá alcanzar acuerdos satisfactorios sobre los cuidados que se deben proporcionar en relación a la salud del paciente, así como la responsabilidad de éste en lo que hace al cumplimiento de las indicaciones".

17 "El 'ethos' no es otra cosa que una forma o modo de vida. El fin de la Ética es facilitar el recto actuar de la persona, delimitando la bondad o maldad de los actos. El objetivo de su conocimiento no tiene como finalidad saber qué es la virtud, lo cual no tendría ninguna utilidad, sino llegar a ser virtuoso".

18 El Senado de Argentina sancionó la Ley 26.529 de 2009 (Congreso de la República Argentina, 2009, p. 26), "Derechos del Paciente en su Relación con los Profesionales e Instituciones de la Salud". Es una ley congruente con el Código de Ética, breve y concisa que caracteriza los derechos del paciente, la historia clínica y el Cl. El Código Argentino fundamenta filosóficamente los temas y remite a la ley cuando es pertinente.

19 El Artículo 77 señala: "El paciente tiene derecho a que se le brinde la información que permita obtener su consentimiento comprensivo del diagnóstico, pronóstico, terapéutica y cuidados preventivos primarios o secundarios correspondientes a su estado de salud. Deberá firmar él, la familia o su representante un libre 'Consentimiento Informado' cuando los facultativos lo consideren necesario".

20 Artículo 138, "el trabajo en equipo no exime a quien lo dirige o a quienes tengan funciones asignadas, de cumplir con el libre Consentimiento Informado, cuyas características en algunos procedimientos llegan más allá de la firma de un formulario preestablecido".

21 "Artículo 248, "los datos médicos recolectados no deben proporcionarse ni divulgarse a terceros sin el consentimiento expreso 
En síntesis, en referencia al $\mathrm{Cl}$, y a otros temas de igual importancia, el código argentino es el más completo, claro y sólido de los aquí revisados, tanto en lo filosófico, como en lo ético y en lo procedimental.

\section{Discusión}

\section{Del paternalismo al reduccionismo de la autonomía}

Aunque el respeto profesado por la autonomía del ciudadano es constitutivo de la sociedad moderna, la "democratización de las decisiones sanitarias" (Gracia, 2002) es un desarrollo relativamente reciente. Sólo hasta la segunda mitad del siglo pasado los conceptos de dignidad humana y autogestión del cuerpo logran permear a la sociedad y al ejercicio sanitario, confiriéndole a la condición de autonomía la preponderancia que previamente le había sido otorgada al principio de beneficencia.

Adicionalmente, desde finales del siglo XIX, emergen múltiples denuncias sobre el ejercicio médico que motivan cambios en la protección legal de los pacientes y erosionan la confianza que la sociedad depositaba en la profesión ${ }^{22}$. Así, la relación clínica, antaño cimentada en un pacto primario de confianza, fue sustituida por la sospecha.

En consecuencia, experimentamos una "desidealización patológica" del paternalismo, en donde éste dejó de ser un acto benévolo para convertirse en algo perverso y agresivo (Zuleta, 2015) ${ }^{23}$. Producto de ello, germinan las declaraciones de derechos de los pacientes y surge el Cl. Este último, también ha evolucionado desde el simple deber de dar información hasta erigirse como un derecho fundamental -enmarcado en la autodeterminación y el libre desarrollo de la personalidad- y en un presupuesto de la lex artis. Ambas propiedades le otorgan su carácter de obligatoriedad (Galán-Cortés, 1999).

De esta manera, al menos en teoría, la relación clínica contemporánea ha modificado el peso de las responsabilidades, tendiendo a equilibrar la balanza. Antes, el adeudo de las decisiones tomadas recaía totalmente sobre el médico, ahora, bajo el arquetipo de la autonomía, el paciente se reconoce -o se idealiza- como un interlocutor válido y, por tanto, tendría también que asumir las obligaciones y consecuencias de su participación. Si bien, el médico y el paciente no cuentan con simetría técnica, el derecho si les otorga una simetría moral, lo que le confiere la legitimidad al acto.

No obstante, el nuevo paradigma de relación médico-paciente ha sido distorsionado en la práctica. Como una desviación del fin del $\mathrm{Cl}$, el modelo de atención en salud ha adoptado compromisos vinculantes que le otorgan una calidad contractual al acto clínico. La propuesta se torna pues propensa al fracaso al contradecir una característica esencial de la medicina, su carácter probabilístico, y al exigir crear certidumbres donde no las hay. Tal desencuentro, entre la promesa de valor y la realidad de la práctica, ha incentivado hechos perniciosos como el creciente número de demandas al sistema sanitario y el consiguiente aumento del gasto en salud producto de la medicina defensiva. Por ejemplo, sólo en los Estados Unidos, el aumento de procedimientos inducidos por el temor al litigio ocasiona un costo estimado entre $\$ 45$ a \$60 billones de dólares al año (Mello, 2010; Kessler, 1996).

de aquellas personas de quienes provienen." Art. 251, "la información proveniente de pacientes que no guarden el anonimato, debe tener el Consentimiento Informado escrito de los mismos".

22 Citando algunos ejemplos de escándalos y abusos en investigación biomédica que motivaron cambios proteccionistas: los crímenes de los campos de concentración de la Alemania nazi; el experimento Tuskeegee (1932-1972); la "catástrofe de la talidomida" (1957 y 1963); los casos de la escuela Willowbrook (1947-1987), etc.

23 "Podemos denominar desidealización patológica al proceso que consiste en convertir el objeto bueno en malo, en lugar de relativizar al primero, situarlo en el conjunto de sus circunstancias, temporalizarlo y pensarlo (...)" (Zuleta, 2015). 
Este fenómeno responde tanto al conocimiento de los nuevos deberes hacia los pacientes, como a la falta de conciencia y coherencia en la asimilación de sus nuevas capacidades, y se debe a que los cambios no han nacido precisamente de la cultura, sino de los tribunales, en donde suelen representarse los intereses y valores individuales sobre los colectivos. Para citar un error común, con frecuencia se permite que se interprete el principio de autonomía del paciente únicamente desde la cara de la responsabilidad del médico, desconociendo de manera inadmisible al otro actor, lo cual constituye una expresión de paternalismo residual. Además, como manifestación de una medicina defensiva, tal enfoque puede promover actitudes nocivas que van desde el abandono o la desprotección del paciente hasta la subordinación médica. De igual manera, favorece demandas arbitrarias y excesivas de los pacientes, que abusan de la permisividad del sistema.

Algunas de las denuncias que esgrimen los pacientes sobre insuficiencia en la información médica son otra muestra de lo expuesto. En condiciones ideales, para considerar legítimo el proceso de interlocución, se requeriría de individuos conscientes de su papel y capacidades en la participación, de pacientes que no se conformasen con la información dada, tendrían también que ser responsables y activos en pedirla. Solo así, el $\mathrm{Cl}$ puede ser una manifestación fidedigna de la moralidad de la del proceso propuesto, de la suficiente ilustración y de la conformidad del mismo, y no un simple acto burocrático. Empero, conocemos las dificultades de llevar a la prácticas este ideal, pues en términos fácticos existirá siempre una brecha de conocimientos entre el médico y su paciente, y un simple proceso informativo nunca conseguirá franquearla por completo. Por todo lo expuesto, aunque el $\mathrm{Cl}$ es un medio para garantizar la declaración de la voluntad del paciente, no es en sí mismo el propósito de la relación ni tiene como alcance hacerla perfecta.

Volviendo a la raíz del concepto, la noción de autonomía en términos kantianos significa auto-obligación (Selbstverpflichtung) y está entendida desde el idealismo de una racionalidad pura. Sin embargo, Kant, en su Fundamentación de la metafísica de las costumbres, establece una característica primordial frecuentemente olvidada: la ética basada en la autonomía del sujeto, no habla de derechos sino de obligaciones, y estas son relacionales. Así pues, el énfasis está en la ley moral, en el deber "razonable", y no en el individuo (Camps, 2011). Posteriormente, en la filosofía secular anglosajona -que nació del liberalismo y se ha fortalecido con los valores del capitalismo, el concepto de autonomía dio un giro hacia la auto-determinación, generando la idea de una sociedad conformada por la articulación de existencias individuales, en donde las personas son totalmente independientes y autosuficientes.

Fue este último marco de referencia conceptual el que impulsó los primeros pasos de la bioética clínica y, aunque en principio su intención era democratizar la relación clínica, promover el respeto por las personas y salvaguardar los intereses de las sociedades pluralistas, hoy nos preguntamos si tal interpretación es un concepto formal o una simple ilusión.

Seguir con el discurso radical de la auto-determinación como el protagonista exclusivo de la asistencia en salud, desligado de otros valores esenciales en las relaciones humanas, como lo son la solidaridad, la empatía, la justicia, el compromiso, la prudencia y sobretodo la confianza, niega un hecho fundamental de nuestra condición: la vulnerabilidad física, psíquica y social que nos caracteriza como seres finitos y en esencia afectivos e interdependientes. Incluso, en el marco de la relación sanitaria, sus actores estamos circunscritos a la dinámica que genera el sistema, la cual está intermediada, burocratizada y fragmentada. 
Tal argumentación no busca para nada justificar el paternalismo, al contrario los mismos postulados demuestran que este es un modelo obsoleto. Solo se quiere visibilizar algunas de las debilidades que el nuevo paradigma debe superar para normalizarse.

Uno de los imperativos del modelo es lograr el empoderamiento de los pacientes, y esta tarea sólo se puede cumplir con el compromiso y trabajo conjunto del Estado y la sociedad. "La auténtica transformación revolucionaria que encierra el concepto de autonomía es la afirmación de una soberanía del individuo sobre sí mismo y sobre su vida, que le permite elegir entre las opciones posibles, haciéndose cargo responsablemente de esa realidad" (Feito-Grande, 2011). En poblaciones con menoscabo de su autonomía, vulnerables por su edad, características sociales, nivel de formación o condiciones orgánicas -como supone algunos estados de enfermedad-, también es deber del sistema sanitario diseñar y promover procedimientos especiales que garanticen las condiciones indispensables para que los pacientes puedan elegir (Garzón, 2009) y contar con medios flexibles de suministro de información para que el consentimiento signifique una intervención real en la decisión (Graham, et al., 2013)

Por otro lado, se requiere sustituir el carácter contractual y biomédico de la relación clínica por lo que realmente es: una relación de compromiso y ayuda, de altísima responsabilidad, inmersa en la confianza y el respeto mutuo (Delgado, 2012). Se requiere también expandir el concepto de respeto hacia el ser en su totalidad. Esto quiere decir que debemos superar el significado reduccionista de la autonomía y comenzar a integrarlo a otros valores que fortalezcan la legitimidad del concepto en el ejercicio sanitario. Es fundamental comprender la naturaleza y el alcance de las relaciones operantes en el cuidado de la salud para lograr los objetivos que justifican esas relaciones y proveer el trato apropiado a los pacientes. Obviamente esta es una tarea que desde el derecho no se podrá solucionar, más si se debe reflexionar desde la sociedad y la academia.

Finalmente, con relación a los profesionales sanitarios, es esencial fortalecer la educación humanística, ética y bioética. Las crisis sociales, políticas y económicas de principios de este siglo han sido, sin duda, crisis éticas, y sin ir muy lejos, las crisis de nuestro sistema sanitario también lo son. Esto demuestra que el enfoque positivista de la Universidad está obsoleto. La formación de valores éticos debe ser un continuo en la vida de los ciudadanos, y por lo tanto, no debe circunscribirse solo al hogar y a los niveles de escolaridad básica. Parafraseando a Victoria Camps, la reflexión sobre la moral lleva siempre a la educación, y la manera de educar es inculcar hábitos. Se trata de crear unos mínimos de costumbres, de maneras de ser y de hacer, que, cuando no existan, creen alarma. El Estado y las universidades deben sumarse a esta preocupación, participar y hacerse responsables y garantes de la formación ética y el perfil moral de sus profesionales.

\section{¿El Proyecto propuesto cumple con su propuesta de valor?}

La respuesta corta seria no. El Proyecto no cumple porque falla desde su ontología. Mientras por qué -la motivación de la iniciativa- está plenamente justificado, el cómo -su método- y en el para qué -las metas propuestas- patinan en proposiciones insuficientes.

La idea de regular el ejercicio de la medicina a través de un nuevo código legislativo, como un mecanismo para proteger los intereses del Estado y controlar el alto impacto que la profesión tiene sobre él -el para qué de la iniciativa-, es una pretensión 
poco realista que evidencia la mirada fútil del problema. Ningún código deontológico por sí mismo va a garantizar el ejercicio ético de sus profesionales ni ayudará a solucionar el statu quo, pues, como se expresó previamente, el fenómeno no es exclusivamente jurídico (Sánchez, 1996). Por otro lado, alinear los intereses del Estado con la profesión habla más de la necesidad de construir una ciudadanía responsable, lo cual también estaría fuera del alcance de cualquier ley.

El código colombiano y el Proyecto de ley tienen como factor diferenciador su robusto desarrollo en temas relativos al funcionamiento de los tribunales de ética. Aunque se requiere establecer claridad legal, llama la atención que, en contraste con otros códigos -como el argentino-, alrededor de la mitad de los artículos del proyecto en curso estén relacionados con aspectos sancionatorios y contenidos conexos. Esto es un problema tanto de forma como de fondo. Más allá de lo punitivo, que pareciera ser el núcleo gravitante, un código deontológico debería manifestar una sólida fundamentación ética y filosófica para formalizar lo adecuado, el deber ser, y dar claridad en aspectos procedimentales, todo esto con el fin de facilitar la interpretación del ejercicio moral, enaltecer la eticidad del quehacer de los profesionales y evitar que su ejercicio se degrade hasta un semblante meramente defensivo. En cambio, el contenido del articulado tiene serias falencias en estos aspectos, es decir, en su esencia, quedando la normatividad ética eclipsada por la jurídica.

El resultado evidente, contrario a lo perseguido, será el fortalecimiento de las conductas defensivas que derivarán en un mayor gasto en salud, sin conseguir restablecer la confianza ni aportar una mayor satisfacción al sistema (Studdert et al., 2005; Kachalia \& Mello, 2013)

\section{Sobre el método, con relación al tema del consentimiento informado.}

El siglo XX culmina fundando un nuevo paradigma de relación médico-paciente, en donde ambos actores interactúan de manera libre y consensuada, participan como sujetos autónomos, con intereses, juicios y valores propios, pero también con responsabilidades claras. Al ser producto de un acuerdo de voluntades, se establece la necesidad de dejar constancia del proceso y se extiende el requerimiento del $\mathrm{Cl}$, sin embargo, su método y alcance aún no han sido bien definidos.

En últimas, el modelo contractual falla al suponer que lo que está escrito se ha dicho y que lo que se ha dicho se ha entendido. Al interpretar el $\mathrm{Cl}$ sólo como un requisito legal, puede reducirse a obtener la firma del paciente en un formato prediseñado, en donde de manera extensa, detallada y defensiva se enumeren todos los posibles riesgos inherentes al procedimiento y al paciente. En cambio, el $\mathrm{Cl}$ debería ser un ejercicio de templanza. Su esencia exige asimilarlo como un proceso hermenéutico que culmina en la manifestación y validación de la autonomía del paciente, hecho necesario para legitimar el acto clínico.

Para ello, el paciente requiere información precisa, particular y a su alcance, ni más ni menos, y la cantidad y suficiencia de esta información dependerá necesariamente de la interlocución entre las dos partes. En este caso, el concepto de "riesgo previsible" patina, ya que su vaguedad deja abierta la imposibilidad de cubrirlo por completo. Basta con mirar el listado de eventos adversos de un medicamento tan común como el acetaminofén para extrapolar la complejidad e incertidumbre que existen en cualquier acto médico. Si bien, infinidad de riesgos pudiesen acontecer en la más mínima intervención, su ocurrencia no necesariamente puede estimarse, incluso esta puede variar en el mismo paciente o entre actos similares, sin que necesaria- 
mente ello incurra en modificaciones sobre las conductas clínicas ni afecte el desenlace. No es posible, y por lo tanto no es ético, exigir a los médicos dar información "exacta" a priori cuando su conocimiento no se mueve en terreno de certezas sino de interpretaciones y probabilidades.

Tampoco es coherente exigir que se emitan todos los posibles efectos, vicisitudes o cursos de las intervenciones -lo que se traduce en una larga enumeración de probabilidades y datos estadísticos-, y a la vez garantizar que está información no va a generar emotividad o angustia al paciente. Es común que los pacientes ingresen a sus valoraciones médicas argumentando preocupaciones exaltadas por estos documentos. El miedo que se genera incluso hace desistir temporalmente a algunos, quienes pierden oportunidad en el tratamiento, con el subsecuente incremento del riesgo. Por otro lado, la predicción de la respuesta emocional es un aspecto muy difícil de ponderar en los pacientes, estando incluso ajeno a la profesión misma, pues además de la capacidad cognitiva que permite la comprensión del discurso, un juicio responsable y prudente requiere la capacidad de gobernar racionalmente la consideración afectiva y asumir responsabilidades sobre riesgos y beneficios derivados de la toma de decisiones. Para llevar esto a la práctica clínica se requeriría superar grandes vacíos formativos y replantear el diseño de atención sanitaria, comenzando, por ejemplo, con contar con mucho más tiempo para la valoración de los pacientes, volver al concepto de médico de cabecera y formar a los profesionales en habilidades y competencias propias de la psicología, entre otros.

Más que el deber de informar todos los riesgos que pudieran preverse, lo que el paciente debería conocer son las características generales de los procesos a los que va a ser sometido, los riesgos esperados que en él podrían tener impacto sobre su salud o calidad de vida, y los que van a inducir modificaciones en su atención o en la toma de decisiones clínicas, dejando claro que esto no representa un estimativo perfecto, sino un ejercicio que se hace desde el juicio médico con la información que le aporta el paciente y que busca tomar acciones para disminuir la incertidumbre de los procesos clínicos. Empero, para ser coherentes con el paradigma de la autonomía, debería manifestarse de forma explícita que la suficiencia en la información no la determina exclusivamente el médico, y el paciente tiene el derecho y el deber de solicitar cualquier información que desee.

Finalmente, el documento de $\mathrm{Cl}$ pudiese sólo entenderse como una "virtualidad legal de la autonomía" (Moore, Gómez, \& Kurtz, 2012), pues lo importante no es el papel en sí, sino el acto moral que le precede (Kottow, 2007).

En su ejecución debe quedar claro que la medicina es una profesión de medios, basada en la competencia profesional y la responsabilidad de permanente autorregulación ${ }^{24}$, y no ofrece resultados perfectos. "Por cuanto la medicina está demasiado plagada de incertidumbres, probabilidades y efectos inesperados que no pueden ser plasmados en un documento" (Kottow, 2007, p. 31). La promesa de valor del médico solo puede ir hasta su obrar dentro de la lex artis y su compromiso de respeto, veracidad, acompañamiento y cuidado al paciente, entendiendo además que su participación esta engranada en un sistema que también aporta capacidades y restricciones.

24 Como lo señala el art. 104 de la Ley 1438, parágrafo 5: "Las actividades profesionales y la conducta de los profesionales de la salud debe estar dentro de los límites de los códigos de ética profesional vigentes. Las asociaciones científicas deben alentar a los profesionales a adoptar conductas éticas para mayor beneficio de sus pacientes". 


\section{Conclusión}

El Cl proviene de un vacío ético dejado por la profesión médica que ha sido ocupado por el derecho positivo occidental. Aunque fue establecido como un marco de protección a la autonomía de los pacientes, el reconocimiento de este derecho no será posible sólo por el hecho de que sea ordenado por una herramienta jurídica. En el ámbito sanitario es menester que florezcan prácticas éticas, y para que esto se haga realidad, la norma debe ser asumida desde la cultura misma de sus profesionales. Además, se requiere un proceso de formación y empoderamiento social que debe constituirse como una tarea compartida del Estado, los administradores, los trabajadores de los servicios de salud, las universidades y la ciudadanía en general.

Aunque se ratifica la necesidad de promulgar un nuevo código de ética médica en Colombia, para que este código encarne los fines de la medicina moderna y de cuenta de la sociedad democrática, laica y plural que representa, requiere reformular sus principios, siendo además claro y suficiente en lo procedimental. Por tanto, en su desarrollo necesita contar con la participación de la academia en extenso e integrar a los diferentes actores en su discurso.

Algunos de los aspectos necesarios para elaborar códigos de ética médica ya han sido contemplados en otras latitudes. Se considera apropiado el enfoque argentino de formular un código en extenso, que incluya a todo el equipo que participa en la atención de los pacientes. Es razonable postular que lo mismo puede hacerse en Colombia, en donde el sistema sanitario se ve afectado por las acciones e interacciones de diferentes actores.

\section{Agradecimientos:}

A Manuela, a ANESTESIAR sindicato, al equipo de la maestría en Bioética de la Universidad CES y a Olga Yanneth Cubides Moreno.

\section{Referencias}

Asociación Médica Argentina. (2011). Código de Ética para el Equipo de Salud. Segunda edición corregida y aumentada. Recuperado 15 de octubre de 2016, a partir de https:// www.ama-med.org.ar/images/uploads/files/ama-codigo-etica-castellano.pdf

Camps V. (2011). La autonomía, el principio «por defecto». Bioètica \& Debat, 17(62), 11-13. https://dialnet.unirioja.es/servlet/articulo?codigo $=3678217$

Cañete R, Guilhem D, Brito K. (2012). Consentimiento informado: algunas consideraciones actuales, 18(1), 121-127. http://www.scielo.cl/scielo.php?script=sci arttext\&pid=S1726-569X2012000100011

Colegio Médico Chileno. (2011). Código de Ética del Colegio Médico de Chile A.G. Recuperado 14 de octubre de 2016, a partir de http://www.colegiomedico.cl/ wp-content/uploads/2015/06/colmed codigo etica 2013.pdf

Confucio. (s. f.). Capítulo 17.3. En Las Analectas (p. 72). Alejandría digital. Recuperado a partir de http://www.alejandriadigital.com/wp-content/uploads/2015/12/ CONFUCIO-Analectas.pdf

Congreso de la República Argentina. (2009). Ley 26.529: Derechos del Paciente en su Relación con los Profesionales e Instituciones de la Salud. Recuperado 16 
de octubre de 2016, a partir de http://servicios.infoleg.gob.ar/infoleglnternet/ anexos/160000-164999/160432/norma.htm

Congreso de la República de Colombia. (1981). Ley 23 de 1981. Por la cual se dictan normas en materia de ética médica. Recuperado 10 de octubre de 2016, a partir de http://www.archivogeneral.gov.co/sites/all/themes/nevia/PDF/Transparencia/ LEY 23 DE_1981.pdf

Delgado, J. (2012). Nuevas perspectivas bioéticas: Autonomía relacional. ENE. Revista de Enfermería, 6(1), 36-43. http://ene-enfermeria.org/ojs/index.php/ENE/article

Feito-Grande L. (2011). El concepto de autonomía en la medicina occidental. Bioètica \& Debat, 17(62), 1-6. http://www.raco.cat/index.php/BioeticaDebat_es/article/ viewArticle/257005

Galán-Cortés, JC. (1999). La responsabilidad médica y el consentimiento informado. Rev Med Uruguay, 15, 5-12. http://www.rmu.org.uy/revista/1999v1/art2.pdf

Garzón F. (2009). Aspectos bioéticos del consentimiento informado en investigación biomédica con población vulnerable. Revista latinoamericana de bioetica, 9(2), 8-27. http://www.redalyc.org/pdf/1270/127020306002.pdf

Gracia D. (2002). De la bioética clínica a la bioética global: Treinta años de evolución. Acta Bioética, 8(1), 27-39. http://www.scielo.cl/scielo.php?script=sci arttext\&pid=S1726-569X2002000100004

Gracia D. (2004a). El Juramento Hipocrático. En Gracia D (Ed.), Como arqueros al blanco, Estudios de bioética (Primera edición, pp. 245-264). Madrid: Triacastela. http:// www.scielo.cl/scielo.php?script=sci arttext\&pid=S0717-92272006000300008

Gracia D. (2004b). La ética y las profesiones sanitarias. En Gracia D (Ed.), Como arqueros al blanco, Estudios de bioética (Primera edición, pp.265-278). Madrid:Triacastela.http:// www.scielo.cl/scielo.php?script=sci arttext\&pid=S0717-92272006000300008

Graham A, Powell M, Taylor N, Anderson D, \& Fitzgerald R. (2013). Investigación ética con niños (Primera edición). Florencia: Centro de investigaciones de UNICEF - Innocenti. http://childethics.com/wp-content/uploads/2015/04/ERIC-compendium-ES_LR.pdf

Kachalia A, \& Mello MM. (2013). Defensive Medicine. Legally Necessary but Ethically Wrong? Inpatient Stress Testing for Chest Pain in Low-Risk Patients. JAMA, 173(12), 1056-7. https://www.ncbi.nlm.nih.gov/pubmed/23689540

Kessler D, \& McClellan M. (1996). Do doctors practice defensive medicine? Q J Econ, 111(2), 353-90. https://academic.oup.com/qje/article-abstract/111/2/353/1938365/Do

Kottow M. (2007). Consentimiento informado: aspectos generales. En Kottow M (Ed.), Participación informada en clínica e investigación biomédica: Las múltiples facetas de la decisión y el consentimiento informados (Primera edición, pp. 82-8). Bogotá: UNESCO y Universidad Nacional de Colombia. http://www.unesco.org.uy/shs/ fileadmin/templates/shs/archivos/ 
Kottow M. (2007). Participación informada en clínica e investigación biomédica: las múltiples facetas de la decisión y el consentimiento informados. Bogotá. http:// www.unesco.org.uy/shs/fileadmin/templates/shs/archivos

Mello MM, Chandra A, Gawande AA, \& Studdert DM. (2010). National costs of the medical liability system. Health affairs, 29(9), 1569-77. https://www.ncbi.nlm. nih.gov/pmc/articles/PMC3048809/

Moore, P., Gómez, G., \& Kurtz, S. (2012). Comunicación médico-paciente: una de las competencias básicas pero diferentes. Atención Primaria, 44(6), 358-365. https:// doi.org/10.1016/j.aprim.2011.07.008

Ocampo-Martínez J. (2002). La bioética y la relación médico-paciente. Cir Ciruj, 70(1), 55-59. http://www.medigraphic.com/pdfs/circir/cc-2002/cc021k.pdf

Organización Médica Colegial de España. (2011). Código de Deontología Médica español. Recuperado 15 de octubre de 2016, a partir de https://www.cgcom.es/ sites/default/files/codigo deontologia medica.pdf

Pinto D, \& Gulfo R. (2013). Asentimiento y consentimiento informado en pediatría: aspectos bioéticos y jurídicos en el contexto colombiano. Revista colombiana de bioética, 8, 144-165. http://www.bioeticaunbosque.edu.co/publicaciones/Revista/rev81/arti9 Borispinto.pdf

Sánchez-González MA. (1996). El Consentimiento Informado, un derecho del enfermo. Cuadernos del Programa Regional de Bioética. Organización Panamericana de la Salud. Recuperado 15 de noviembre de 2016, a partir de http://www. colmed2.org.ar/images/code02.pdf

Senado de la República de Colombia. (2015). Informe de ponencia para primer debate al Proyecto de Ley 24/2015: por medio de la cual se crea el nuevo Código de Ética Médica. En Gaceta del Congreso, año XXIV - № 755 (Vol. XXIV-755, pp. 1-24). Bogotá: Imprenta Nacional de Colombia. http://www.imprenta.gov.co/gacetap/ gaceta. mostrar documento?p tipo=22\&p numero $=256 \& p$ consec $=44607$

Senado de la República de Colombia, 2015. Texto definitivo aprobado en sesión plenaria al proyecto de ley 24 de 2015, por medio de la cual se crea el nuevo Código de Ética Médica. Congreso de la República de Colombia (14 de diciembre de 2016). Recuperado el 17 de marzo de 2017 a partir de: http://www.imprenta.gov. co/gacetap/gaceta.mostrar documento?p tipo=1037\&p numero=24\&p con$\underline{\mathrm{sec}=47088)}$

Studdert DM, Mello MM, Sage WM, DesRoches CM, Peugh J, Zapert K, \& Brennan TA. (2005). Defensive medicine among high-risk specialist physicians in a volatile malpractice environment. JAMA, 293(21), 2609-17. https://www.ncbi.nlm.nih. gov/pubmed/15928282

Zuleta E. (2015). Idealización en la vida personal y colectiva. En Elogio de la dificultad y otros ensayos. Editorial Planeta Colombiana S.A. http://static0.planetadelibros. com.co/libros contenido extra/31/30482 1 Elogio zuleta.pdf 